\title{
Algorithm for controlling parameters of linear filter based on fuzzy expert system
}

\author{
Alexandr Eliseev ${ }^{1, *}$, Alexandr Prygunov ${ }^{1}$ \\ ${ }^{1}$ Don State Technical University, 344000 Gagarin square, 1, Rostov-on-Don, Russia
}

\begin{abstract}
The problem of linear filtration of parameters of the vector of an maneuvering object state is considered. The problems to detect the maneuver and control the filter coefficients are solved. A fuzzy expert system is used to adapt the filter. The efficiency of the filter is shown in the test case.
\end{abstract}

\section{Introduction}

In the organization of traffic of civil aircrafts the control authorities widely use trajectory information coming from the radio surveillance equipment. The surveillance equipment intended for monitoring the position and movement parameters of aircraft include the multipurpose radar systems, automatic dependent surveillance equipment, and multilateration systems. One of the most important parameters characterizing the quality of a radar is the error in estimating the parameters of the aircraft trajectory, especially under the conditions of maneuvering. This error depends on many factors, including the methods and algorithms used for the secondary processing of the radar measurements. In the secondary processing system the dynamic filtering algorithms with maneuver detection are widely used [125]. These algorithms consist of three main modules: maneuver detection module; module for adjusting filter parameters; and filter module $[1,2]$. The existing variety of dynamic filtering algorithms is due to the features of the implementation of these modules. For example paper [6] proposes an algorithm for adjusting the Kalman filter by changing the value of the elements of the matrix of the intensities of the forming noises. However, the feature of this algorithm is the iterative adjustment, which leads to the dynamic filtering error in the transition period. The purpose of the work is to reduce the dynamic error of the $\alpha-\beta$-filtering of the trajectory parameters of the maneuvering aircraft. The problem to be solved is the development of the algorithm to adjust $\alpha-\beta$-filter parameters

\section{Mathematical formulation of the problem}

Let the parameters of the trajectory of the maneuvering aircraft on the time interval $\left[t_{0}, T\right]$ be described by the difference equation $[1,2]$ :

$$
\mathbf{X}\left(t_{j+1}\right)=\left\{\begin{array}{l}
\boldsymbol{\Phi}(j+1, j) \mathbf{X}(j)+\boldsymbol{\Gamma}(j+1, j) \mathbf{N}_{x}(j), \\
t_{j}<t_{B M} ; \\
\boldsymbol{\Phi}(j+1, j) \mathbf{X}(j)+ \\
+\boldsymbol{\Gamma}(j+1, j)\left(\mathbf{A}_{x}(j)+\mathbf{N}_{x}(j)\right), \\
t_{B M} \leq t_{j}<t_{E M} ; \\
\boldsymbol{\Phi}(j+1, j) \mathbf{X}(j)+\boldsymbol{\Gamma}(j+1, j) \mathbf{N}_{x}(j), \\
t_{j} \geq t_{E M},
\end{array}\right.
$$

and the observation equation has the form

$$
\mathbf{Z}(j)=\mathbf{H}(j) \mathbf{X}(j)+\mathbf{N}_{z}(j), j=0,1,2,
$$

where $\mathbf{X}(j)$ is the vector of the process parameters; $\mathbf{Z}(j)$ is the measurement vector; $\boldsymbol{\Phi}(j+1, j)$, $\boldsymbol{\Gamma}(j+1, j), \mathbf{H}(j)$ are known functional matrices; $t_{\mathrm{BM}}$

, $t_{\mathrm{EM}}$ are the start and end time of the maneuver respectively; $\mathbf{A}_{x}(j)$ is the vector of the intensity of the process (maneuver) changes with unknown elements; $\mathbf{N}_{x}(j), \mathbf{N}_{z}(j)$ are random noises of the object (1).

It is required: to obtain according to the results of current observations $\mathbf{Z}(j)$ the optimal (in terms of the mean-square) estimate $\hat{\mathbf{X}}(j)$ of the filtration of the state vector (1) under conditions of a priori uncertainty with

*Corresponding author: eliseev_av65@mail.ru 
respect to the values of the elements of the matrix $\mathbf{A}_{x}(j)$.

\section{Solution to the problem}

To improve the speed of the estimation $\hat{\mathbf{X}}(j)$ of the parameters vector and to reduce the requirements for the amount of memory and performance of the digital computer complex of the subsystem for secondary processing of trajectory measurements, we will consider the $\alpha-\beta$-filter as tracking filter [1]:

$$
\begin{aligned}
& \hat{\mathbf{X}}(j+1)=\hat{\mathbf{X}}(j+1 \mid j)+ \\
& +\mathbf{K}(j+1)[\mathbf{Z}(j+1)-\mathbf{H}(j+1) \hat{\mathbf{X}}(j+1 \mid j)],
\end{aligned}
$$

where $\hat{\mathbf{X}}(j+1 \mid j)=\boldsymbol{\Phi}(j+1, j) \hat{\mathbf{X}}(j)$,

$$
\mathbf{K}(j)=\operatorname{diag}\left[\mathbf{K}_{X i}(j), i=\overline{1, q}\right],
$$

$\mathbf{K}_{X i}(j)=\left[\alpha_{i}(j), \beta_{i}(j) / \Delta T\right]^{T}, \Delta T=t_{j}-t_{j-1}$ is the time interval between measurements $\mathbf{Z}(j)$, $j=1,2,3, \ldots, \alpha_{i}(j), \beta_{i}(j)$ are elements of the matrix of the amplification coefficients $\mathbf{K}_{X i}(j), q$ is the dimensionality of the state vector $\hat{\mathbf{X}}(j)$.

In the general case, when the state vector of a nonobjectifying object is filtered the coefficients $\alpha_{i}(j)$, $\beta_{i}(j)$ vary according to the following law [1]:

$$
\alpha_{i}(j)=\frac{2(2 j-1)}{j(j+1)}, \beta_{i}(j)=\alpha_{i}^{2}(j) /\left(2-\alpha_{i}(j)\right) .
$$

Thus, in order to adapt the $\alpha-\beta$-filter, it is necessary to solve consistently two main problems: to detect the beginning or ending of the maneuver and to control the values of the amplification coefficient elements $\mathbf{K}(j)$.

Let us consider successively the possible solutions to these problems.

The problem to detect a maneuver. To solve this problem, it is necessary to enter a maneuver detector (MD) $[1,2]$ in the adaptive $\alpha-\beta$-filter, which should form a maneuver sign $S M\left(t_{j}\right)=S M(j) \in\{0,1\}$ that assumes a value of " 1 " in case of detecting the beginning of maneuver (continuation of maneuver), or " 0 " in case of the maneuver end (lack of maneuver).

Let us introduce, by analogy with [6], two additional characteristics of residual: the modulus of the arithmetic mean of the residual $|\bar{\varepsilon}(j)|$, and the arithmetic mean of the modulus of the residual value $\overline{|\varepsilon(j)|}$ (for simplicity, without loss of generality, we assume that the residual is scalar)

$$
\begin{array}{r}
|\bar{\varepsilon}(j)|=\frac{\left|\sum_{i=0}^{n-1} \varepsilon(j-i)\right|}{n}, \\
\overline{|\varepsilon(j)|}=\frac{\sum_{i=0}^{n-1}|\varepsilon(j-i)|}{n}
\end{array}
$$

where $n$ is the number of consecutive subsequent measurements used to detect maneuvering, $\boldsymbol{\varepsilon}(j)=\mathbf{Z}(j)-\mathbf{H}(j) \hat{\mathbf{X}}(j \mid j-1)$.

The criterion for detecting a maneuver, taking (4) into account, takes the form

$$
K_{\mathrm{OM}} \equiv\left\{\begin{array}{c}
(S M=1), \text { if }|\bar{\varepsilon}(j)|=\overline{|\varepsilon(j)|} \\
(S M=0), \text { if }|\bar{\varepsilon}(j)|<\overline{|\varepsilon(j)|}
\end{array}\right.
$$

The problem to control the values of the amplification coefficient elements $\mathbf{K}(j)$. To adapt the $\alpha-\beta$-filter to change of the model of the movement of an object we use the operatively advising expert system (OAES), which is based on the application of fuzzy logic inference [26-28]. We will assume that the process of the filter functioning can be represented in the form of a tuple of some problem situations (PrS). Any PrS can be described by a situational vector $\mathbf{s v}=\left[s v_{k}, k=\overline{1, K}\right]^{T}$, each coordinate of which $s v_{k}$ is a linguistic variable with a given set of terms $\left\{S V_{k}^{l}, l=\overline{1, m_{k}}\right\}$. We think that for some concrete realizations of the situational vector $\mathbf{s v}^{*}$ there are precedents of the successful solution of the current PrS, characterized by some precedent vector $\mathbf{p v}=\left\{p v_{m}, m=\overline{1, m_{P V}}\right\}$, each coordinate $p v_{m}$ of which is a linguistic variable with a given set of terms $\left\{P V_{m}^{p}, p=\overline{1, n_{m}}\right\}$. Suppose that some PrS, which occurs during the dynamic filtering of measurements, can be described by a situational vector $\mathbf{s v}=\left[s v_{k}, k=\overline{1,2}\right]^{T}$ with the following elements: $s v_{2}-$ "the value of the relative error module $|\delta \varepsilon(j)|$ ", where $|\delta \varepsilon(j)|$ is determined by the formula

$$
|\delta \varepsilon(j)|=\frac{\sum_{i=0}^{n-1}|\varepsilon(j-i)|}{n \sigma^{*}},
$$

where $\sigma^{*}$ is some allowable mean-square error $\varepsilon(j)$

Let these variables be described by the following term sets: $\mathbf{S V}_{1}=\{$ "PE", "IDE" $\}=\left\{S V_{1}^{l}, l=\overline{1,2}\right\}$; 


$$
\mathbf{S V}_{2}=\left\{\begin{array}{l}
\text { "VSE","SE","AE", } \\
\text { "BE","VBE" }
\end{array}\right\}=\left\{S V_{2}^{l}, l=\overline{1,5}\right\},
$$

where "PE", "IDE" are the terms "permissible error" and "invalid dynamic error", respectively, "VSE" is "very small error", "SE" is "small error", "AE" is "average error", "BE" is "large error", "VBE" is "very large error".

We assume that the linguistic variable $s v_{1}$ is given on a unified universal set $E=\{0,1\}$, the linguistic variable $s v_{2}$ on a universe $E=\left[0,\left|\delta \varepsilon_{\max }\right|\right]$, and their terms are described by membership functions $\mu_{S V_{k}} \in\left\{\mu_{S V_{k}^{l}}, l=\overline{1, m_{k}}\right\}$.

Let the terms of a linguistic variable $s v_{1}$ be described by membership functions of the singleton type:

$$
\begin{aligned}
S V_{1}^{1}: \mu_{S V_{1}^{1}} & =\left\{\begin{array}{l}
1, \text { if } x=\bar{x}_{1}^{1}=0-" P E^{\prime \prime} ; \\
0, \text { если } x \neq \bar{x}_{1}^{1} ;
\end{array}\right. \\
S V_{1}^{2}: \mu_{S V_{1}^{2}} & =\left\{\begin{array}{l}
1, \text { if } x=\bar{x}_{1}^{2}=1-" I D E ; \\
0, \text { if } x \neq \bar{x}_{1}^{2} .
\end{array}\right.
\end{aligned}
$$

The terms of the linguistic variable $s v_{2}$ are described by the following membership functions [3]:

the terms $S V_{2}^{1}, S V_{2}^{5}$ :

$$
\mu_{S V_{2}^{l}}(|\delta \varepsilon|)=1-\frac{1}{1+\exp \left(-a_{2 l}\left(|\delta \varepsilon|-c_{2 l}\right)\right)}, l \in\{1,5\},(6)
$$

the terms $S V_{2}^{2}, S V_{2}^{3}, S V_{2}^{4}$ :

$$
\mu_{S V_{2}^{l}}(|\delta \varepsilon|)=\exp \left(\frac{-\left(|\delta \varepsilon|-c_{2 l}\right)^{2}}{a_{2 l}}\right), l \in\{2,3,4\},
$$

where $a_{2 l}, c_{2 l}, l=\overline{1,5}$ are the membership functions parameters. Taking into account that the controlled parameter of the $\alpha-\beta$-filter, as it was said before, is the matrix of amplification coefficients $\mathbf{K}(j)$, and in the particular scalar case it is the coefficients $\alpha(j), \beta(j)$, we introduce a precedent vector $\mathbf{P V}=\left\{p v_{m}, m=\overline{1, m_{P V}}\right\}=\left\{p v_{1}\right\}$ consisting of one variable $p v_{1}-$ "value of the coefficient $\alpha(j)$ ". In addition we assume that the type of the precedent vector is determined by the value of the element of the situation vector $s v_{1}$ - "filtering error type".

Thus when $s v_{1}=$ "PE", the value $\alpha(j)$ is determined based on the following precedent

$$
p v_{1}: \alpha(j)=\left\{\begin{array}{l}
0.743-0.043 j, \text { при } j \leq 8, \\
0.4 \text { при } j>8 .
\end{array}\right.
$$

In the case when $s v_{1}$ takes the linguistic meaning "invalid dynamic error", the precedent vector contains a linguistic variable $p v_{1}$ specified on the universe $E=[0,1]$, and its terms are described by membership functions $\mu_{P V_{1}} \in\left\{\mu_{P V_{1}^{p}}, p=\overline{1, n_{m}}\right\}$.

Let the linguistic variable $p v_{1}$ be described by the following term sets:

$$
\mathbf{P V}_{1}=\left\{\begin{array}{l}
\text { VSV } \alpha ", " \mathrm{SV} \alpha ", " \mathrm{AV} \alpha ", \\
\text { "BV } \alpha \text { ", "VBV } \alpha "
\end{array}\right\}=\left\{P V_{1}^{p}, p=\overline{1,5}\right\},
$$

where "VSV $\alpha$ " is "very small value of the parameter $\alpha(j)$ ", "SV $\alpha$ " is "small value of the parameter $\alpha(j)$ ", "AV $\alpha$ " is "average value of the parameter $\alpha(j)$ ", "BV $\alpha$ " is "large value of the parameter $\alpha(j)$ ", "VBV $\alpha$ " is "very large value of the parameter $\alpha(j)$ ".

We define the terms of the set $\mathbf{P} \mathbf{V}_{1}$ by membership functions similarly to (6), (7).

The system of rules describing the mechanism for solving the current $\mathrm{PrS}$ arising during the operation of the $\alpha-\beta$-filter takes the form:

$$
\begin{gathered}
r=1 \text { : if }\left(s v_{1}=" P E^{\prime \prime}\left(\mu_{S V_{1}^{1}}\right)\right) \text {, then }\left(p v_{1}=\left\{\begin{array}{l}
0.743-0.043 j \forall j \leq 8, \\
0.4 \forall j>8
\end{array}\right),\right. \\
r=2: \text { if }\left(s v_{1}=" I D E^{\prime \prime}\left(\mu_{S V_{1}^{2}}\right)\right) \text { and }\left(s v_{2}=" V S E^{\prime \prime}\left(\mu_{S V_{2}^{1}}\right)\right) \text {, then }\left(p v_{1}=" V S V \alpha^{\prime \prime}\left(\mu_{P V_{1}^{1}}\right)\right), \\
r=3 \text { : if }\left(s v_{1}=" I D E^{\prime \prime}\left(\mu_{S V_{1}^{2}}\right)\right) \text { and }\left(s v_{2}=" S E^{\prime \prime}\left(\mu_{S V_{2}^{2}}\right)\right) \text {, then }\left(p v_{1}=" S V \alpha^{\prime \prime}\left(\mu_{P V_{1}^{2}}\right)\right), \\
r=4: \text { if }\left(s v_{1}=" I D E^{\prime \prime}\left(\mu_{S V_{1}^{2}}\right)\right) \text { and }\left(s v_{2}=" A E^{\prime \prime}\left(\mu_{S V_{2}^{3}}\right)\right) \text {, then }\left(p v_{1}=" A V \alpha^{\prime \prime}\left(\mu_{P V_{1}^{3}}\right)\right), \\
r=5 \text { : if }\left(s v_{1}=" I D E^{\prime \prime}\left(\mu_{S V_{1}}\right)\right) \text { and }\left(s v_{2}=" B E^{\prime \prime}\left(\mu_{S V_{2}^{4}}\right)\right) \text {, then }\left(p v_{1}=" B V \alpha^{\prime \prime}\left(\mu_{P V_{1}^{4}}\right)\right), \\
r=6 \text { : if }\left(s v_{1}=" I D E^{\prime \prime}\left(\mu_{S V_{1}^{2}}\right)\right) \text { and }\left(s v_{2}=" V B E^{\prime \prime}\left(\mu_{S V_{2}^{5}}\right)\right) \text {, then }\left(p v_{1}=" V B V \alpha^{\prime \prime}\left(\mu_{P V_{1}^{5}}\right)\right) \text {. }
\end{gathered}
$$


Using the "minimax" Mamdani-Zade rule [26-28], the expression for calculating the final membership function $\mu_{p v_{1 \Sigma}}$ of the output linguistic variable $p v_{1}$ can be written as:

$\mu_{p v_{I \Sigma}}\left(s v_{1}^{*}, s v_{2}^{*}\right)=\max _{r} \min _{p v_{1}}\left(\min _{k}\left(\mu_{S V_{k}(r)}^{*}\right), \mu_{P V_{1}(r)}\right)$,

where, $\mu_{S V_{k}(r)} \in\left\{\mu_{S V_{k}^{l}}, l=\overline{1, m_{k}}\right\}$,

$\mu_{P V_{1}(r)} \in\left\{\mu_{P V_{1}^{p}}, p=\overline{1, n_{m}}\right\}$ are the membership functions, respectively, of the linguistic variables $s v_{k}$ and $p v_{1}$, which are part of the production rule with the number $r$, $s v_{1}^{*}, s v_{2}^{*}$ are clear values of the input variables, $\mu_{S V_{k}(r)}^{*}=\mu_{S V_{k}(r)}\left(s v_{k}^{*}\right)$. To find the clear value $p v_{1}^{*}$ of the output variable, we use the "center of gravity" method [26].

To assess the effectiveness of the developed algorithm, the mathematical simulation was carried out. We use the following model of aircraft movement:

$$
\begin{gathered}
\mathbf{X}(j+1)=\left\{\begin{array}{l}
\boldsymbol{\Phi} \mathbf{X}(j)+\boldsymbol{\Gamma} n_{x}(j),(0 \leq j<100) \vee \\
\vee(200 \leq j<400) \vee(500 \leq j \leq 600) ; \\
\mathbf{\Phi} \mathbf{X}(j)+\boldsymbol{\Gamma}\left(1.5 g+n_{x}(j)\right), 100 \leq j<200 ; \\
\boldsymbol{\Phi} \mathbf{X}(j)+\boldsymbol{\Gamma}\left(-4 g+n_{x}(j)\right), 400 \leq j<500,
\end{array}\right. \\
\mathbf{\Phi}=\left[\begin{array}{cc}
1 & \tau \\
0 & 1
\end{array}\right], \boldsymbol{\Gamma}=\left[\begin{array}{c}
\tau^{2} \\
2 \\
\tau
\end{array}\right], \\
\tau=t_{j+1}-t_{j}=1 \mathrm{c}, \quad j=\overline{0,600} .
\end{gathered}
$$

As a result of the simulation it was established that the application of the developed adaptation algorithm allows to reduce the dynamic filtering error by $35 \%$ in comparison with the classical $\alpha-\beta$-filter.

\section{References}

1. A. Farina, F. Studer, Radio and communications, (1993)

2. H.W. Sorenson, Kalman filtering: theory and application (IEEE Press, New York 1985)

3. P. Zarchan, Fundamentals of Kalman filtering - $A$ practical approach (American Institute of Aeronautics and Astronautics, USA 2005)

4. B.P. Gibbs, Advanced Kalman filtering, leastsquares and modeling: a practical handbook, (Hoboken: John Wiley \& Son, Canada 2011)

5. I.R Petersen., A.V. Savkin, Robust Kalman filtering for signals and systems with large uncertainties. (Birkhauser, Boston 1999)

6. M. Rodkin, In Proc. VI All-Russian Conference Radiolocation and radio communication, 125-128, (2012) [in Russian]
7. Y.G. Bulychev., A.V. Eliseev, Avtomatika i Vychislitel'naya Tekhnika, no.2, 26-35, (1998) [in Russian]

8. A.V. Eliseev, Aviakosmicheskoe priborostroenie, 3038, (2006) [in Russian]

9. A.V. Yeliseyev, Radioelectronics and Communications Systems, 13-19 (2005) [in Russian]

10. K.V. Ramachandra, Kalman filtering techniques for radar tracking, (Marcel Dekker, New York 2000)

11. V. Gomez, A. Maravall, Approximate Kalman filtering. (World Scientific, Singapore 1993)

12. M. Verhaegen, P. Van Dooren, IEEE Transactions on Automatic Control, no.10, 907-917, (1986)

13. Q. Xia, M. Rao, Y. Ying, X. Shen, Automatica, no.8, 1333-1338 (1994)

14. Y. Geng, J. Wang, Journal of Beijing University of Aeronautics \& Astronautics, no.5, 434-437, (2004)

15. M. Oussalah, J. Schutter, In Proc. 25th Int. Conf. on Noise and Vibration Engineering, 1225-1232 (2000)

16. R. A. Singer, IEEE Transactions on Aerospace and Electronic Systems, no.4, 473-483 (1970)

17. T. Simeonova, T. Semerdjiev, Inform. \& Security. No.9, 154-165 (2002)

18. R. A. Best., J. P. Norton, IEEE Transactions on Aerospace and Electronic Systems, no.3, 1030-1037 (1997)

19. T.C. Wang, P. K. Varshney, IEEE Transactions on Aerospace and Electronic Systems, no.3,910-924 (1993)

20. Y.T. Chan, F. Couture, IEEE Proc. on Radar and Signal Processing, 1, 21-28 (1993)

21. Y. Bar-Shalom, K. Birmiwal, IEEE Trans. Aerosp. Electron. Syst., no.5, 621-629 (1982)

22. H.B. Sui, X.Y. Fang, and Y. Wu, Radar Science and Technology, no.3, 202-205 (2008)

23. F.M. Sun, X.Q. Wu, K. Qi, Journal of Data Acquisition \& Processing, no.2, 191-195 (2008)

24. X.R, Li,. V.P. Jilkov, IEEE Trans. Aerosp. Electron. Syst., no.1, 96-119 (2010)

25. X.R. Li,. V.P. Jilkov, In Proc. Signal and Data Processing of Small Targets, 6-8 (2010).

26. V. Gostev, Designing fuzzy logic controls for automatic control systems (BHV-Petersburg, $\mathrm{SPb}$ 2011)

27. A.V. Eliseev, Aviatsionnaya Tekhnika, no.3, 20-25, (2005)

28. A.V. Eliseev, Avtomatizatsiya i Sovremennye Tekhnologii, no.11, 3-12, (2005) 